\title{
Band Structure and Magneto- Transport Properties in II-VI Nanostructures Semiconductors - Application to Infrared Detector Superlattices
}

\author{
Abdelhakim Nafidi \\ Laboratory of Condensed Matter Physics and Nanomaterials for Renewable Energy \\ University Ibn-Zohr, \\ Agadir \\ Morocco
}

\section{Introduction}

To observe phenomena by the image through invisible radiation to the eye is probably doubtless an old dream of humanity. The way followed to observe the invisible is to study an artificial retina constituted by a matrix of elements sensitive to radiation and to associate it with a system of acquisition and data processing. In brief a camera or a digital camera sensitive to other radiations than the visible light. The detectors are constituted of simple elementary $\mathrm{p}-\mathrm{n}$ junctions realized in a semiconductor material whose height band gap is adapted to the energy of infrared photons which we wish to detect.

The work of (Essaki \& Tsu, 1970) caused a big interest to the study of superlattices made from alternating layers of two semiconductors. The development of molecular beam epitaxy (MBE) was successfully applied to fabricate different quantum wells and superlattices. Among them III-V superlattices [Ga1-xAl $\mathrm{Gl}_{x} \mathrm{As}-\mathrm{GaAs}$ (Essaki \& Tsu, 1970) - (Dingle et al., 1975) - type I], IV-IV [InAs/GaSb (Sakaki et al, 1978) - type II] and later II-IV superlattice [HgTe/CdTe (Bastard, 1982) - type III]. The later is a stable alternative for application in infrared optoelectronic devices than the $\mathrm{Hg}_{1-\mathrm{x}} \mathrm{Cd}_{\mathrm{x}} \mathrm{Te}$ alloys. Especially in the region of second atmospheric window (around $10 \mu \mathrm{m}$ ) which is of great interest for communication.

$\mathrm{HgTe}$ and CdTe crystallize in zinc -blend lattice respectively. The lattice-matching within $0.3 \%$ yield to a small interdiffusion between $\mathrm{HgTe}$ and CdTe layers at low temperature near $200{ }^{\circ} \mathrm{C}$ by MBE. HgTe is a zero gap semiconductor (due to the inversion of relative positions of $\Gamma_{6}$ and $\Gamma_{8}$ edges (Tuchendler et al, 1973)) when it is sandwiched between the wide gap semiconductor CdTe $(1.6 \mathrm{eV}$ at $4.2 \mathrm{~K})$ layers yield to a small gap HgTe/CdTe superlattice which is the key of an infrared detector.

A number of papers have been published devoted to the band structure of this system (Bastard, 1982) as well as its magnetooptical and transport properties (Nafidi, 2002). The aim of this work is to show the correlation between calculated bands structures and magnetotransport properties in two $\mathrm{HgTe} / \mathrm{CdTe}$ nanostructures superlattices. 
Our calculations of the specters of energy $E\left(d_{2}\right), E\left(k_{z}\right)$ and $E\left(k_{p}\right)$, respectively, in the direction of growth and in plane of the superlattice;were performed in the envelope function formalism. Our two samples are denoted SL1 and SL2.

In the SL1, X-ray diffraction, conductivity, Hall effect, Seebek and Shubnikov-de Haas effects and angular dependence of the transverse magnetoresistance were measured. The profile of the (222) Bragg reflection indicated a modulated structure. At $4.2 \mathrm{~K}$, the sample exhibits $\mathrm{p}$ type conductivity with a Hall mobility of $8200 \mathrm{~cm}^{2} / \mathrm{Vs}$. This allowed us to observe the Shubnikov-de Haas effect with $\mathrm{p}=1,8010^{12} \mathrm{~cm}^{-2}$. Using the calculated effective mass $\left(\mathrm{m}^{*} \mathrm{HH}=0,297 \mathrm{~m}_{0}\right)$ of the degenerated heavy holes gas, the Fermi energy (2D) was $E_{\mathrm{F}}=14 \mathrm{meV}$ in agreement with $12 \mathrm{meV}$ of thermoelectric power $\alpha$. In intrinsic regime, $\alpha \sim T-3 / 2$ and $R_{H}$ $\mathrm{T}^{3 / 2}$ indicates a gap $\mathrm{E}_{\mathrm{g}}=\mathrm{E}_{1}-\mathrm{HH}_{1}=190 \mathrm{meV}$ in agreement with calculated $\mathrm{E}_{\mathrm{g}}(\Gamma, 300 \mathrm{~K})=178$ $\mathrm{meV}$. The formalism used here predicts that the system is semiconductor for $d_{1} / d_{2}=1,87$ and $\mathrm{d}_{2}<150 \AA$. Here, $\mathrm{d}_{2}=30 \AA$ and $\mathrm{E}_{\mathrm{g}}(\Gamma, 4.2 \mathrm{~K})=111 \mathrm{meV}$ so this sample is a twodimensional modulated nanostructure medium-infrared detector semiconductor $(7 \mu \mathrm{m}<\lambda<11 \mu \mathrm{m})$.

In the SL2, the angular dependence of the transverse magnetoresistance follows the twodimensional (2D) behaviour with Shubnikov-de Haas oscillations. While the hall voltage goes to zero when the field is parallel to the plane. At low temperature, the sample exhibits p type conductivity with a hole mobility of $900 \mathrm{~cm}^{2} /$ V.s. A reversal the sign of the weakfield Hall coefficient occurs at $25 \mathrm{~K}$ with an electron mobility of $3.10^{4} \mathrm{~cm}^{2} / \mathrm{Vs}$. In intrinsic regime, the measured $\mathrm{E}_{\mathrm{g}} \approx 38 \mathrm{meV}$ agree with calculated $\mathrm{E}_{\mathrm{g}}(\Gamma, 300 \mathrm{~K})=34 \mathrm{meV}$ witch coincide with the Fermi level energy. The formalism used here predicts that the system is semi metallic for $\mathrm{d}_{1} / \mathrm{d}_{2}=4.1$ and $\mathrm{d}_{2} \geq 30 \AA$. In our case $\mathrm{d}_{2}=44 \AA$ and the gap $\mathrm{E}_{\mathrm{g}}(\Gamma, 4.2 \mathrm{~K})=3 \mathrm{meV}$. In spite of it, this sample exhibits a semi metallic $p$ type conduction mechanism, with a quasi-two-dimensional behavior and is a far-infrared detector $(50 \mu \mathrm{m}<\lambda<450 \mu \mathrm{m})$. The investigated thicknesses of the SL2 situate it at the semiconductor to semimetal electronic transition.

In conclusion, we will show that the $\mathrm{HgTe} / \mathrm{CdTe}$ nano-superlattice is a stable alternative for application in infrared optoelectronic devices than the alloys $\mathrm{Hg}_{1-\mathrm{x}} \mathrm{Cd}_{\mathrm{x}} \mathrm{Te}$.

\section{Experimental techniques}

Our two HgTe/CdTe superlattices samples SL1 and SL2, grown by molecular beam epitaxy (MBE) on a CdTe (111) substrate at $180{ }^{\circ} \mathrm{C}$, had a period $\mathrm{d}=\mathrm{d}_{1}+\mathrm{d}_{2}$ (90 layers) of $\mathrm{HgTe}\left(\mathrm{d}_{1}=5,6\right.$ $\mathrm{nm}) / \operatorname{CdTe}\left(\mathrm{d}_{2}=3 \mathrm{~nm}\right)$ and (100 layers) of $\operatorname{HgTe}\left(\mathrm{d}_{1}=18 \mathrm{~nm}\right) / \operatorname{CdTe}\left(\mathrm{d}_{2}=4.4 \mathrm{~nm}\right)$ respectively. Each sample was cut from an epitaxial wafer with a typical size of $5 \times 1 \times 1 \mathrm{~mm}^{3}$. The ohmic contacts were obtained by chemical deposition of gold from a solution of tetrachloroauric acid in methanol after a proper masking to form the Hall crossbar. Carriers transport properties were studied in the temperature range $1.5-300 \mathrm{~K}$ in magnetic field up to 17 Tesla. Conductivity, Hall Effect, Seebek effect, X-ray diffraction and angular dependence of the transverse magnetoresistance were measured. The measurements at weak magnetic fields (up to $1.2 \mathrm{~T}$ ) were performed into standard cryostat equipment. The measurements of the magnetoresistance were done under a higher magnetic field (up to $17 \mathrm{~T}$ ), the samples were immersed in a liquid helium bath, in the center of a superconducting coil. Rotating samples with respect to the magnetic field direction allowed one to study the angular dependence of the magnetoresistance. 


\section{Theory of structural bands}

Calculations of the spectra of energy $E\left(k_{z}\right)$ and $E\left(k_{p}\right)$, respectively, in the direction of growth and in plane of the superlattice; were performed in the envelope function formalism (Bastard, 1982) (Nafidi, 2002) with a valence band offset $\Lambda$ between heavy holes bands edges of $\mathrm{HgTe}$ and CdTe of $40 \mathrm{meV}$ determined by the magneto-optical absorption experiments (Guldner, 1984)

The general dispersion relation of the light particle (electron and light hole) subbands of the superlattice is given by the expression (Bastard, 1982) :

$$
\cos \mathrm{k}_{\mathrm{z}}\left(\mathrm{d}_{1}+\mathrm{d}_{2}\right)=\cos \left(\mathrm{k}_{1} \mathrm{~d}_{1}\right) \cos \left(\mathrm{k}_{2} \mathrm{~d}_{2}\right)-\frac{1}{2}\left[\left(\xi+\frac{1}{\xi}\right)+\frac{\mathrm{k}_{\mathrm{p}}{ }^{2}}{4 \mathrm{k}_{1} \mathrm{k}_{2}}\left(\mathrm{r}+\frac{1}{\mathrm{r}}-2\right)\right] \sin \left(\mathrm{k}_{1} \mathrm{~d}_{1}\right) \sin \left(\mathrm{k}_{2} \mathrm{~d}_{2}\right)
$$

where the subscripts 1 and 2 refer to HgTe and CdTe respectively. $k_{z}$ is the superlattice wave vector in the direction parallel to the growth axis $\mathrm{z}$. The two-dimensional wave vector $\mathrm{k}_{\mathrm{p}}\left(\mathrm{k}_{\mathrm{x}}, \mathrm{k}_{\mathrm{y}}\right)$ describes the motion of particles perpendicular to $\mathrm{k}_{\mathrm{z}}$. Here,

$$
\xi=\frac{\mathrm{k}_{1}}{\mathrm{k}_{2}} \mathrm{r} \text {, and } \mathrm{r}=\frac{\mathrm{E}-\varepsilon_{2}}{\mathrm{E}+\left|\varepsilon_{1}\right|-\Lambda}
$$

$\mathrm{E}$ is the energy of the light particle in the superlattice measured from the top of the $\Gamma_{8}$ valence band of bulk CdTe, while $\varepsilon \mathrm{i}(\mathrm{i}=1$ or 2$)$ is the interaction band gaps $\mathrm{E}\left(\Gamma_{6}\right)-\mathrm{E}\left(\Gamma_{8}\right)$ in the bulk $\mathrm{HgTe}$ and CdTe respectively. At given energy, the two-band Kane model (Kane, 1957).gives the wave vector $\left(\mathrm{k}_{\mathrm{i}}{ }^{2}+\mathrm{k}_{\mathrm{p}}{ }^{2}\right)$ in each host material:

$$
\left.\begin{array}{ll}
\frac{2}{3} \mathrm{P}^{2} \hbar^{2}\left(\mathrm{k}_{1}{ }^{2}+\mathrm{k}_{\mathrm{p}}{ }^{2}\right)=(\mathrm{E}-\Lambda)\left(\mathrm{E}-\Lambda+\left|\varepsilon_{1}\right|\right) & \text { for HgTe } \\
\frac{2}{3} \mathrm{P}^{2} \hbar^{2}\left(\mathrm{k}_{2}{ }^{2}+\mathrm{k}_{\mathrm{p}}{ }^{2}\right)=\mathrm{E}\left(\mathrm{E}-\varepsilon_{2}\right) & \text { for CdTe }
\end{array}\right\}
$$

$\mathrm{P}$ is the Kane matrix element given by the relation:

$$
\frac{2 \mathrm{P}^{2}}{3\left|\varepsilon_{1}\right|}=\frac{1}{2 \mathrm{~m}^{*}}
$$

where $\mathrm{m}^{*}=0.03 \mathrm{~m}_{0}$ is the electron cyclotron mass in HgTe (Tuchendler et al, 1973). For a given energy E, a superlattice state exists if the right-hand side of Eq. (1) lies in the range $[-1,1]$. That implies $-\pi / d \leq k_{z} \leq-\pi / d$ in the first Brillion zone.

The heavy hole subbands of the superlattice are given by the same Eq. (1) with :

$$
\xi=\frac{\mathrm{k}_{1}}{\mathrm{k}_{2}} \mathrm{r} \text {, and } \mathrm{r}=1 \quad \text { with } \begin{cases}-\frac{\hbar^{2}\left(\mathrm{k}_{1}{ }^{2+} \mathrm{k}_{\mathrm{p}}{ }^{2}\right)}{2 \mathrm{~m}^{*} \mathrm{HH}}=\mathrm{E}-\Lambda & \text { for HgTe } \\ -\frac{\hbar^{2}\left(\mathrm{k}_{2}^{2}+\mathrm{k}_{\mathrm{p}}{ }^{2}\right)}{2 \mathrm{~m}^{*} \mathrm{HH}}=\mathrm{E} & \text { for CdTe }\end{cases}
$$


$\mathrm{m}^{*} \mathrm{HH}=0.3 \mathrm{~m}_{0}$ (Tuchendler et al, 1973) is the effective heavy hole mass in the host materials. The band structure computation consists of solving Eq. (1) which represents the dispersion relations (i.e. finding the values of energy E which are roots of the Eq. (1) for a given value of the carrier wave vector). Here, we are interested in studying the states of energy of light particles and heavy holes in $\mathrm{HgTe} / \mathrm{CdTe}$ superlattice as function of $\mathrm{k}_{\mathrm{z}}$ when $\mathrm{k}_{\mathrm{p}}=0$ and as function of $k_{p}$ when $k_{z}=0$ and when $k_{z}=\pi / d$. The solving procedure used for studying $E$ as function $k_{z}$ in the case where $k_{p}=0$ consists of going, with a steep $\delta E$, through the studied range of energy $E$ and then finding, for each value of $E$, the value of $k_{z}$ which satisfies the dispersion relations. The same procedure is used for studying $E$ as function $k_{p}$ in the case where $k_{z}=0$ and $k_{z}=\pi / d$. It is noteworthy that, for a given value of E, Eq. (1) may have more than one root in $\mathrm{k}_{\mathrm{p}}$. It appears, from Eq. (3)-(5), that the carrier wave vectors $\mathrm{k}_{1}, \mathrm{k}_{2}$, and $\mathrm{k}_{\mathrm{p}}$ are either real or imaginary (i.e. complex) and then using complex numbers in the calculation seems to be more adequate.

\section{The SL1: $\mathrm{HgTe}\left(\mathrm{d}_{1}=5,6 \mathrm{~nm}\right) / \mathrm{CdTe}\left(\mathrm{d}_{2}=3 \mathrm{~nm}\right)$}

\subsection{Theoretical results and discussions}

The energy $E$ as a function of $\mathrm{d}_{2}$, at $4.2 \mathrm{~K}$, in the center $\Gamma$ of the first Brillouin zone and for our $d_{1}=1.87 d_{2}$, is shown in Fig. 1. (a) (El Abidi et al, 2010). The case of our sample $\left(d_{2}=30\right.$ $\AA$ ) is indicated by the vertical broken line. Here the cross-over of $\mathrm{E}_{1}$ and $\mathrm{HH}_{1}$ subbands occurs. $d_{2}$ controls the superlattice band gap $E_{g}=E_{1}-E_{H H 1}$. For weak $d_{2}$ the sample is semiconductor with a strong coupling between the HgTe wells as in the SL1. At the dot $\mathrm{T}\left(\mathrm{d}_{2}=150 \AA \mathrm{A}, \mathrm{E}=39 \mathrm{meV}\right)$ the gap goes to zero with the transition semiconductor- semimetal. When $d_{2}$ increases, $E_{1}$ and $h_{1}$ states drops in the energy gap $[0, \Lambda]$ and become interface state with energy

$$
\mathrm{E}_{\mathrm{I}}=\frac{\Lambda \varepsilon_{2}}{\left|\varepsilon_{1}\right|+\varepsilon_{2}}=34 \mathrm{meV}
$$

for infinite $d_{2}$ obtained from Eq. (1). Then the superlattice has the tendency to become a layer group of isolated HgTe wells and thus assumes a semimetallic character. The ratio $d_{1} / d_{2}$ governs the width of superlattice subbands (i.e. the electron effective mass). A big $d_{1} / d_{2}$, of 4.09 as in the case of the SL2, moves away the material from the two-dimensional behavior.

In the Fig. 1. (b) we can see that the band gap $\mathrm{E}_{\mathrm{g}}(\Gamma)$ increases, presents a maximum at 40 $\mathrm{meV}$ and decreases when the valence band offset $\Lambda$ between heavy hole band edges of $\mathrm{HgTe}$ and CdTe increase. For each $\Lambda, \mathrm{E}_{\mathrm{g}}(\Gamma)$ increases with $\mathrm{T}$. Our chosen value of $40 \mathrm{meV}$ is indicated by a vertical dashed line. This offset agrees well with our experimental results contrary to $0 \mathrm{meV}$ used by (Bastard, 1982) and $360 \mathrm{meV}$ given by (Johnson et al, 1988). The later offset give $\mathrm{E}_{\mathrm{g}}(300 \mathrm{~K})=135 \mathrm{meV}$ in the right of Fig. 1 whereas, in intrinsic regime, $\alpha \sim \mathrm{T}^{-}$ $3 / 2$ and $\mathrm{R}_{\mathrm{H}} \mathrm{T}^{3 / 2}$ indicates a measured gap $\mathrm{E}_{\mathrm{g}} \approx 190 \mathrm{meV}$ in agreement with calculated $\mathrm{E}_{\mathrm{g}}(\Gamma, 300$ $\mathrm{K})=178 \mathrm{meV}$.

Fig. 2 shows that, for each $d_{2}, E_{g}(\Gamma)$ decreases when $d_{1} / d_{2}$ increases. For each $d_{1} / d_{2}$, when $d_{2}$ increases $E_{g}(\Gamma)$ decreases, go to zero at the transition dot $T$ and became negative for a semimetal conductivity. In the right of Fig. 2, the cut-off wavelength $\left|\lambda_{c}\right|$ diverge at $T$ with

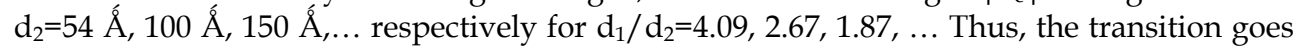
to high $d_{2}$ when $d_{1} / d_{2}$ decreases. In the case of our samples the transition occurs, respectively, at $\mathrm{d}_{2}=150 \AA \hat{\AA}$ and $54 \AA$ in the SL1 and the SL2. 

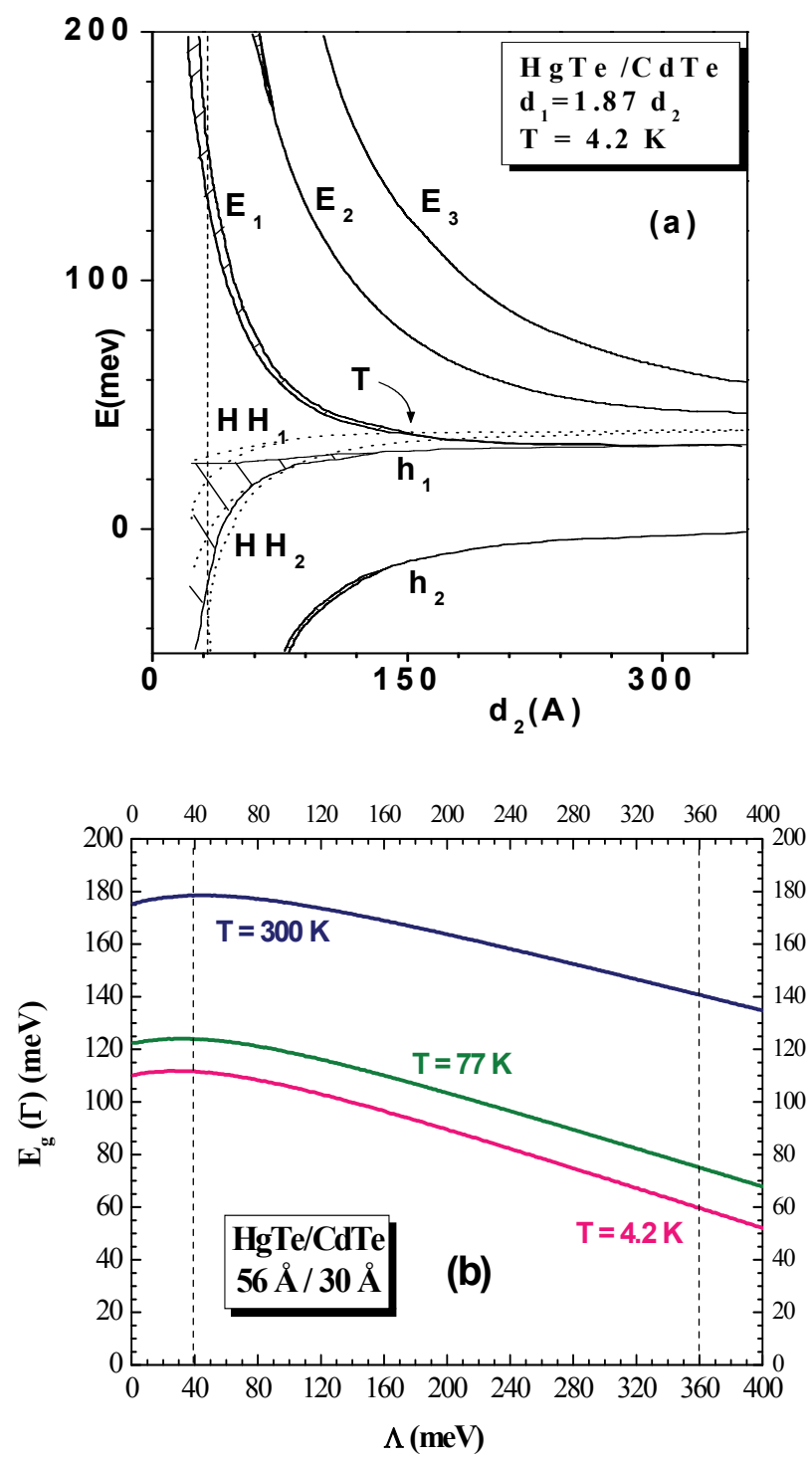

Fig. 1. (a) Energy position and width of the conduction $\left(\mathrm{E}_{\mathrm{i}}\right)$, heavy-hole $\left(\mathrm{HH}_{\mathrm{i}}\right)$, and the lighthole $\left(h_{1}\right)$ subbands calculated at $4,2 \mathrm{~K}$ in the center $\Gamma$ of the first Brillouin zone as a function of layer thickness $\mathrm{d}_{2}$ for HgTe/CdTe superlattice with $\mathrm{d}_{1}=1.87 \mathrm{~d}_{2}$, where $\mathrm{d}_{1}$ and $\mathrm{d}_{2}$ are the thicknesses of the HgTe and CdTe layers, respectively. $\mathrm{T}$ is the point of the transition semiconductor- semimetal. (b) the band gap $\mathrm{E}_{\mathrm{g}}(\Gamma)$, in the center $\Gamma$ of the first Brillouin zone, as function of temperature and valence band offset $\Lambda$ between heavy holes bands edges of $\mathrm{HgTe}$ and CdTe for the investigated $\mathrm{HgTe} / \mathrm{CdTe}$ superlattice 


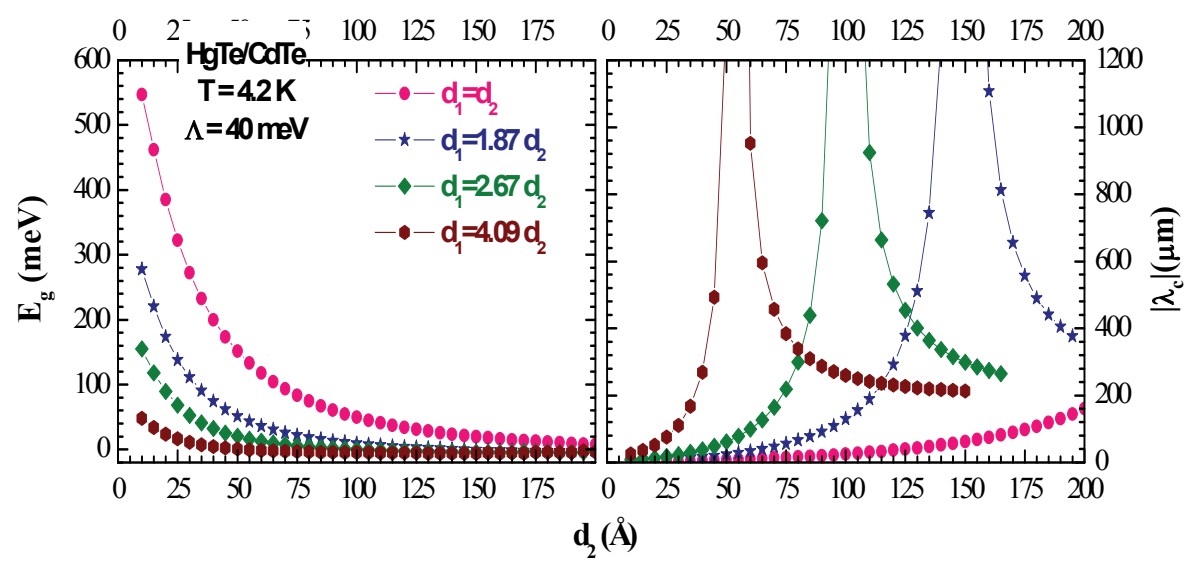

Fig. 2. $E_{g}(\Gamma)$ and $|\lambda c|$ as function of $d_{2}$ for various $d_{1} / d_{2}$ at $4.2 K$

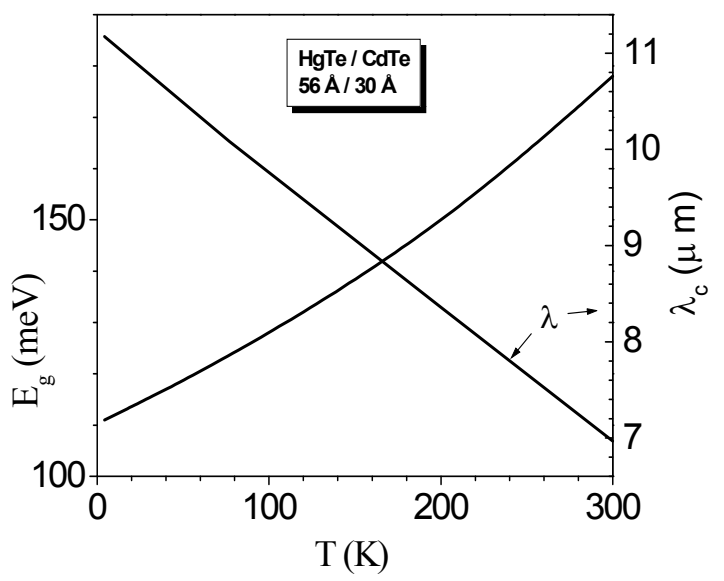

Fig. 3. Temperature dependence of the band gap $E_{g}$ and the cut-off wavelength $\lambda_{c}$, at the center $\Gamma$ of the first Brillouin zone

Using the value of the gaps $\varepsilon_{1}$ and $\varepsilon_{2}$ at different temperatures between $4.2 \mathrm{~K}$ and $300 \mathrm{~K}$ (Weiler, 1981) and taking $\mathrm{P}$ temperature independent, this is supported by the fact that from Eq. (4) $\mathrm{P} \approx \varepsilon_{\mathrm{i}}(\mathrm{T}) / \mathrm{m}^{*}(\mathrm{~T}) \approx$ cte, we get the temperature dependence of the band gap $\mathrm{E}_{\mathrm{g}}$, in the center $\Gamma$ of the first Brillouin zone in Fig. 3. Note that $E_{g}$ increases from $111 \mathrm{meV}$ at $4.2 \mathrm{~K}$ to $178 \mathrm{meV}$ at $300 \mathrm{~K}$. We calculated the detection cut-off wave length by the relation

$$
\lambda_{c}(\mu \mathrm{m})=\frac{1240}{E_{g}(m e V)}
$$


In the investigated temperature range $7 \mu \mathrm{m}<\lambda<11 \mu \mathrm{m}$ situates our sample as a medium infrared detector.

In Fig. 4 we can see the specters of energy $E\left(k_{z}\right)$ and $E\left(k_{p}\right)$, respectively, in the direction of growth and in plane of the superlattice at $4.2 \mathrm{~K}$. Along $\mathrm{k}_{\mathrm{z}}$ the subbands $\mathrm{E}_{1}$ and $\mathrm{h}_{1}$ are wide and non-parabolic. Along $\mathrm{k}_{\mathrm{p}}, \mathrm{E}_{1}$ and $\mathrm{h}_{1}$ increase with $\mathrm{k}_{\mathrm{p}}$ whereas $\mathrm{HH}_{\mathrm{n}}$ decreases. This yield

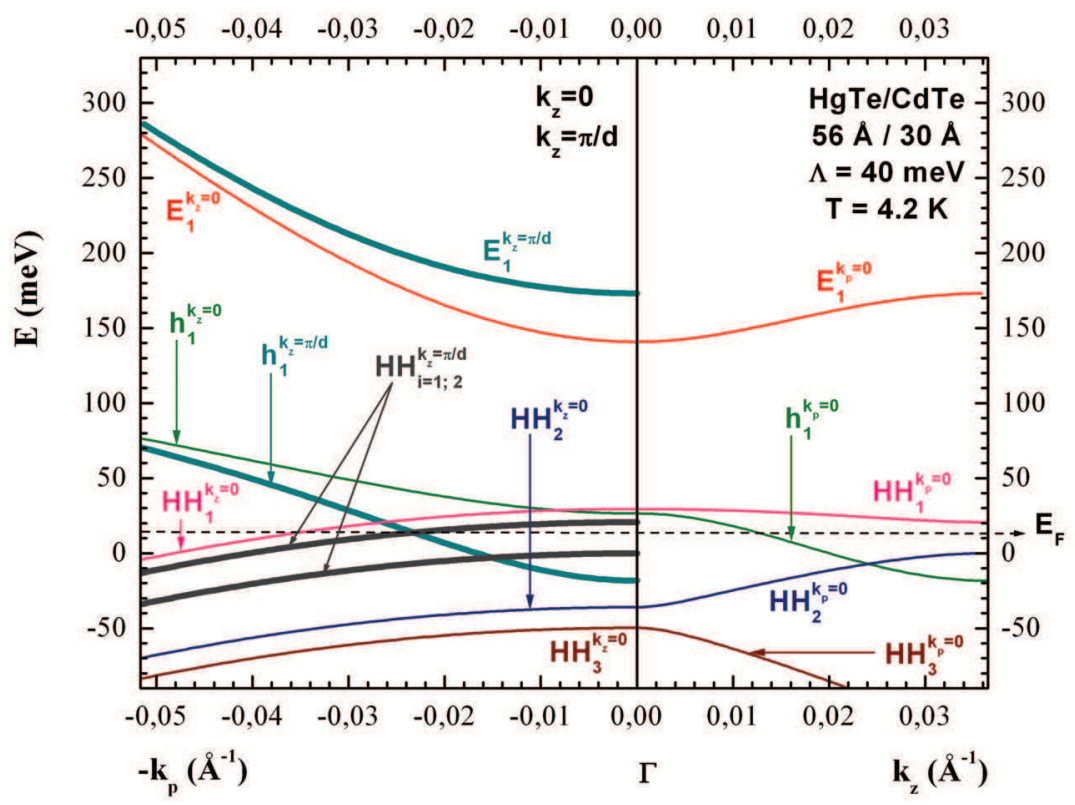

Fig. 4. Calculated bands along the wave vector $k_{z}$ in the right and in plane $k_{p}\left(k_{x}, k_{y}\right)$ in the left, of the $\mathrm{HgTe} / \mathrm{CdTe}$ superlattice at $4.2 \mathrm{~K}$. $\mathrm{E}_{\mathrm{F}}$ is the energy of Fermi level

to an anti-crossing of $\mathrm{HH}_{1}$ and $\mathrm{h}_{1}$ at $\mathrm{k}_{\mathrm{p}}=0.01 \AA^{-1}$ corresponding to a magnetic field of 4 Teslas according to the Formula (13).

\subsection{Experimental results and discussions}

In Fig. 2, $|\lambda c|$ as function of $d_{2}$, at $4.2 \mathrm{~K}$, shows that there is a multiple choice of $\mathrm{d}_{2}$ for medium infrared detectors with $|\lambda c| \leq 10 \mu \mathrm{m}$ for each $d_{1} / d_{2}$. Our choice of $d_{2}=3 n$ m for $\mathrm{d}_{1}=1.87 \mathrm{~d}_{2}$ give a calculated $\mathrm{E}_{\mathrm{g}}(\Gamma, 300 \mathrm{~K})=178 \mathrm{meV}$ corresponding to $\mathrm{E}_{\mathrm{g}}(\Gamma, 300 \mathrm{~K})=183 \mathrm{meV}$ for the well used random alloys $\mathrm{Hg}_{0.8} \mathrm{Cd}_{0.2} \mathrm{Te}$ (Hansen et al, 1982).

In Fig. 5(a) we can see that the angular dependence of the transverse magnetoresistance vanishes, when the field is parallel to the plane of the SL, indicating a two dimensional (2D) behavior confirmed by the observation of SDH oscillations in Fig. 7(a).

At low temperatures, the sample exhibits $p$ type conductivity (confirmed by our thermoelectric power measurements in Fig. 6(b)) with a concentration $\mathrm{p}=1.8410^{12} \mathrm{~cm}^{-2}$ and a Hall mobility $\mu_{p}=8200 \mathrm{~cm}^{2} /$ Vs in Fig. 5(b) (Nafidi et al, 2004). The decrease of $R_{H}(1 / T)$ at $55 \mathrm{~K}$ shown by arrow in Fig. 5 (c) can be due to coupling between HgTe well (small $\mathrm{d}_{1} / \mathrm{d}_{2}$ and $\mathrm{d}_{2}$ ), to the widening of carrier sub-bands under the influence of the magnetic field 

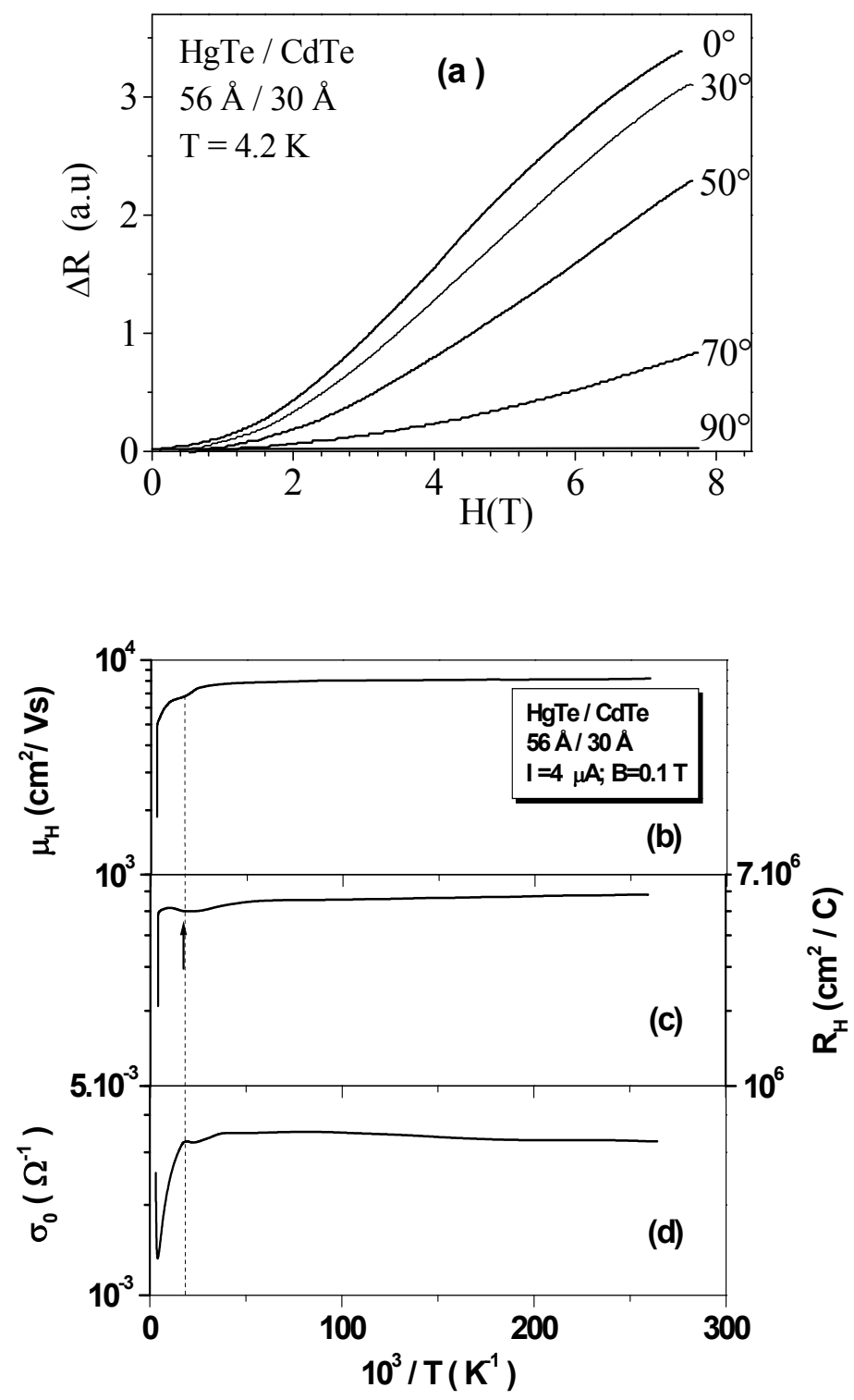

Fig. 5. (a) Variation of magnetoresistance of the sample with various angles between the magnetic field and the normal to the $\mathrm{HgTe} / \mathrm{CdTe}$ superlattice surface. Temperature dependence of the Hall mobility (b), weak-field Hall coefficient (c) and conductivity (d) (Nafidi et al, 2002) in the investigated HgTe/CdTe superlattice 

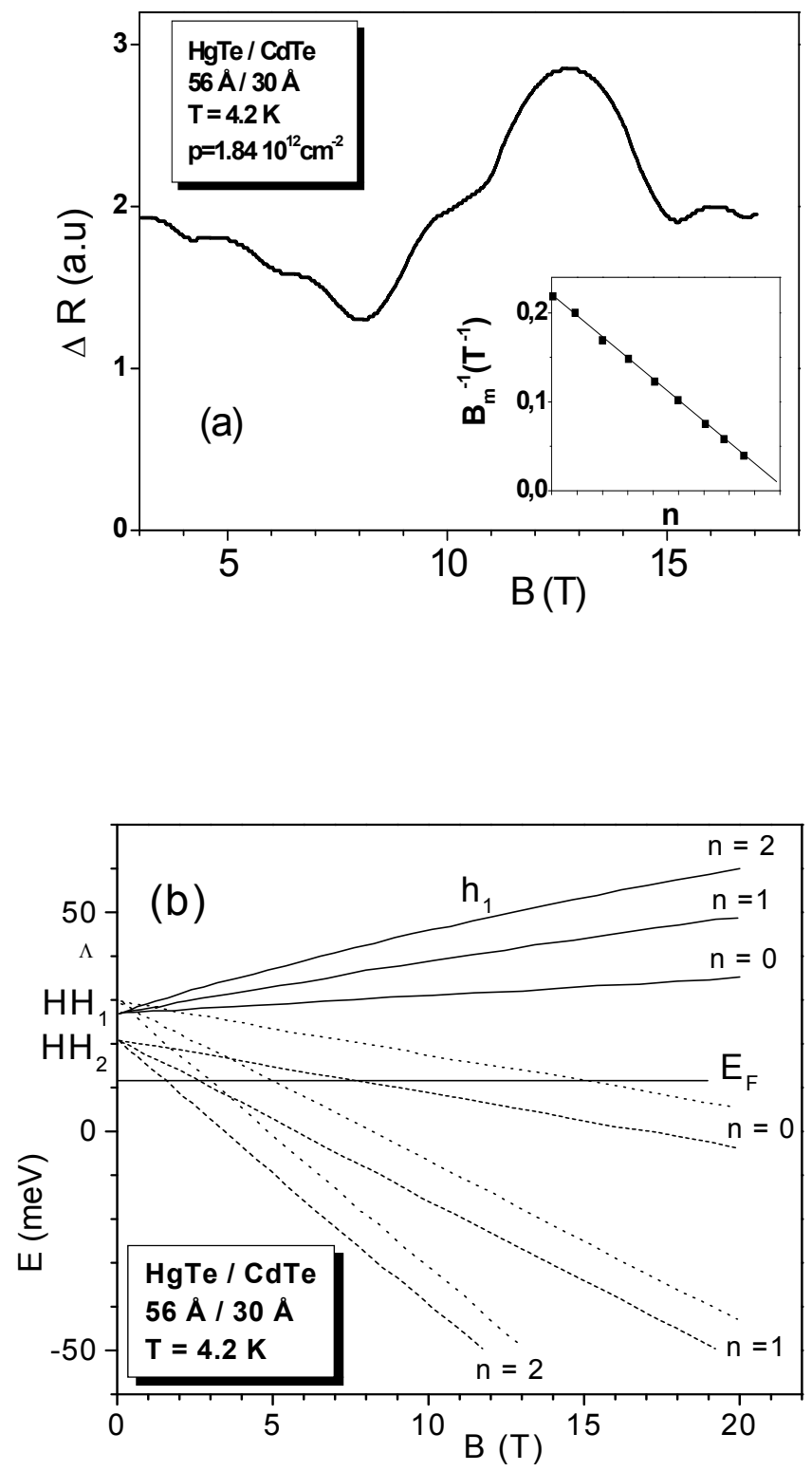

Fig. 6. (a) Variation of transverse magnetoresistance, with magnetic field of $\mathrm{HgTe} / \mathrm{CdTe}$ superlattice, at $4.2 \mathrm{~K}(\mathrm{~b})$ calculated Landau levels as a function of applied magnetic field of the $\mathrm{HgTe} / \mathrm{CdTe} \mathrm{SL}$ at $4,2 \mathrm{~K}$. $\mathrm{E}_{\mathrm{F}}$ is the Fermi level energy 
and/or to the overlap between involved carriers sub-bands $\left(\mathrm{HH}_{1}\right)$ and $\left(\mathrm{h}_{1}\right)$ at $\left(\mathrm{k}_{\mathrm{z}}, \mathrm{k}_{\mathrm{p}}\right)=(0$, $\left.0.008 \AA^{-1}\right)$ and $\left(\pi / d, 0.023 \AA^{-1}\right)$ along $E\left(k_{p}\right)$ in Fig. 4 . Only the later cross over at $E_{F}$ can be taking into account. This increases the concentration $p$ of holes (decreases of $R_{H}(1 / T)$ in Fig. 5 (c)) and decreases of conductivity at $55 \mathrm{~K}$ (in Fig. 5 (d)) yielding to a decrease of mobility (in Fig. 5 (b)) due to the increase of effective mass from $m_{h 1}$ to $m_{H H 1}$. In intrinsic regime (245 $\mathrm{K} \leq \mathrm{T} \leq 300 \mathrm{~K}$ ), the measure of the slope of the curve $\mathrm{R}_{\mathrm{H}} \mathrm{T}^{3 / 2}$ indicates a gap $\mathrm{E}_{\mathrm{g}}=\mathrm{E}_{1}-\mathrm{E}_{\mathrm{HH} 1}=190$ $\mathrm{meV}$ witch agree well with calculated $\mathrm{E}_{\mathrm{g}}(\Gamma, 300 \mathrm{~K})=178 \mathrm{meV}$.

This relatively high mobility $\mu_{\mathrm{p}}=8200 \mathrm{~cm}^{2} / \mathrm{Vs}$ allowed us to observe the Shubnikov-de Haas effect (SDH) until 17 Tesla in Fig. 5. (a) (Nafidi et al, 2006). Its well knows that, for a two dimensional electronic gas, the oscillations of the magnetoresistance is periodic with respect to $1 / \mathrm{B}$. The period $\Delta(1 / \mathrm{B})$ is related to the concentration $\mathrm{p}$ of the holes by the relation:

$$
\mathrm{p}=\frac{\mathrm{e}}{\pi \hbar \Delta\left(\frac{1}{\mathrm{~B}}\right)}
$$

In the insert of Fig. 6(a) we have plot the inverse of the minima's $1 / B_{m}$ as a function of the entire $\mathrm{n}$ following the formula:

$$
\frac{1}{\mathrm{~B}_{\mathrm{m}}}=\Delta\left(\frac{1}{\mathrm{~B}}\right)\left(\mathrm{n}+\frac{1}{2}\right)
$$

The linear line slope gives $\Delta(1 / \mathrm{B})=0.027 \mathrm{~T}^{-1}$ and $\mathrm{p}=1.80 \times 10^{12} \mathrm{~cm}^{-2}($ in good agreement with $1.84 \times 10^{12} \mathrm{~cm}^{-2}$ of weak field Hall effect from Fig. 5 (c)).

At low temperature, the superlattice heavy holes dominate the conduction in plane (Fig 4). The $\mathrm{HH}_{1}$ (and $h_{1}$ ) band is parabolic with respect to $\mathrm{k}_{\mathrm{p}}{ }^{2}$ (from Fig. 4). That permits us to estimate effectives masses $\mathrm{m}_{\mathrm{HH}_{1}}^{*}=0.297 \mathrm{~m}_{0}, \mathrm{~m}_{\mathrm{h}_{1}}^{*}=0.122 \mathrm{~m}_{0}$ and the Fermi energy (2D) at 4.2 K

$$
\left|\mathrm{E}_{\mathrm{F}}-\mathrm{E}_{\mathrm{HH}_{1}}\right|=\left|\frac{\mathrm{p} \pi \hbar^{2}}{\mathrm{~m}_{\mathrm{HH}_{1}}^{*}}\right|=14 \mathrm{meV}
$$

Around the (222) Bragg reflection in Fig. 7(a), a series of steps are observed corresponding to the oscillating counterparts described by (Arch et al, 1986). The difference with our results comes from the monocromacity and the power of their synchrotron source. Our SL1 is a modulated structure with high quality of interfaces.

The thermoelectric power $\alpha$ measurements shown in Fig. 6(b) (Nafidi et al, 2006) indicate a p-type conductivity, confirmed by Hall effect measurements in Fig. 5(c). At low temperature, $\alpha \sim T^{0.8}$ (in the top insert of Fig. 7(b)) is in agreement with Seebeck effect theory deduced from the relaxation time resolution of the Boltzmann equation (Seeger, 2002). For our degenerate holes gas the Seebeck constant is described by the formula:

$$
\alpha=\frac{\left[\left(\pi \mathrm{k}_{\mathrm{B}}\right)^{2} \mathrm{~T}(\mathrm{~s}+1)\right]}{3 \mathrm{eE}_{\mathrm{F}}}
$$



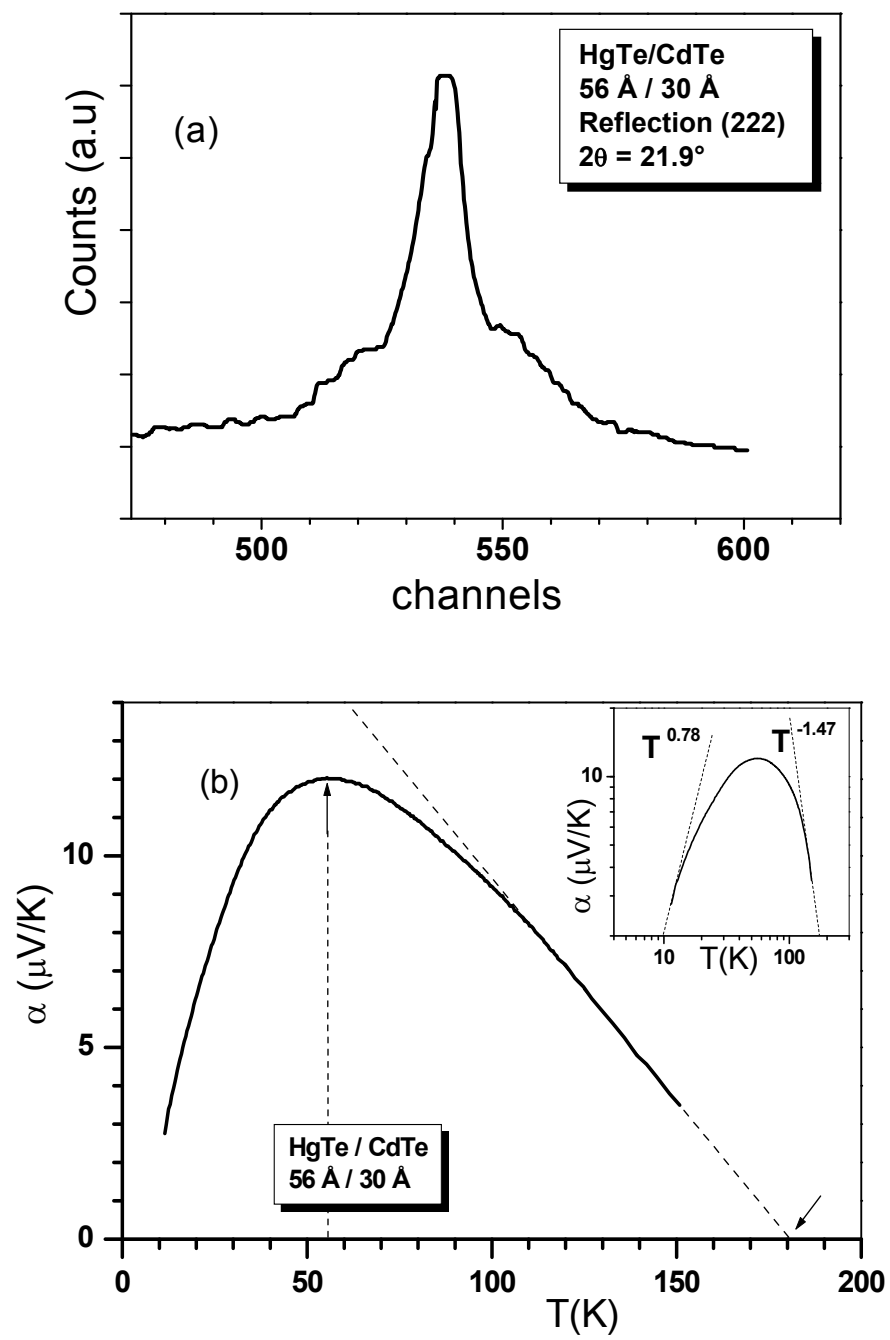

Fig. 7. (a) Room temperature X-ray diffraction profile around the (222) Bragg reflection of the HgTe/CdTe SL. (b) Measured thermoelectric power as a function of temperature of the $\mathrm{HgTe} / \mathrm{CdTe}$ superlattice

and the collision time $\tau \sim \mathrm{E}^{\mathrm{s}-(1 / 2)}$. This permits us to estimate the Fermi energy at $\mathrm{E}_{\mathrm{F}}=12 \mathrm{meV}$ (in Fig. $6(\mathrm{~b})$ ) in agreement with the calculated $\left|\mathrm{E}_{\mathrm{F}}-\mathrm{E}_{\mathrm{HH} 1}\right|=14 \mathrm{meV}$ with $\mathrm{s}=2.06$ corresponding to holes diffusion by ionized impurities. It is relevant here to signal that the maximum of $\alpha$ at $\mathrm{T}=55.2 \mathrm{~K}$ correspond to the shift of Hall mobility in Fig. 5(b). Here $\mu_{\mathrm{HH} 1}=2.434 \mu_{\mathrm{h} 1}$ at $\mathrm{E}_{\mathrm{F}}$. At $\mathrm{T}=190 \mathrm{~K}$, a reversal of $\alpha$ sign will occur corresponding exactly to the minimum of the conductivity $\sigma_{0}$ in Fig. 5(d). From $\mathrm{p}=1.80 \times 10^{12} \mathrm{~cm}^{-2}$ of SDH we have deduced the effective masse of the degenerate heavy holes to be 


$$
\mathrm{m}_{\mathrm{HH}_{1}}^{*}=\frac{\pi \mathrm{p} \hbar^{2}}{\mathrm{E}_{\mathrm{F}}}=0.308 \mathrm{~m}_{0}
$$

(in agreement with the theoretical $\mathrm{m}^{*} \mathrm{HH}_{1}=0.297 \mathrm{~m}_{0}$ ). We calculated the energy of the Landau levels (LL) by transposing the quantification rule of the wave vector in the plane of the SL:

$$
\mathrm{k}_{\mathrm{p}}^{2}=\frac{(2 \mathrm{n}+1) \mathrm{eB}}{\hbar}
$$

where $n$ are the quantum orders of LL. The crossing of $E_{F}$ with LL in Fig. 6(b) indicated the same magnetic field positions as those of the observed SDH oscillations minima $B_{m}$ in Fig. 6(a).

This HgTe/CdTe superlattice is a stable alternative for application in medium infrared optoelectronic devices than the random alloys $\mathrm{Hg}_{0.8} \mathrm{Cd}_{0.2} \mathrm{Te}$ because the small composition $\mathrm{x}=0.22$, with $\mathrm{E}_{\mathrm{g}}(\Gamma, 300 \mathrm{~K})=183 \mathrm{meV}$ given by the empiric formula for $\mathrm{Hg}_{1-\mathrm{x}} \mathrm{Cd}_{\mathrm{x}} \mathrm{Te}$ (Hansen et al, 1982).

$$
E_{g}(x, T)=-0,302+1,93 x-0,810 x^{2}+0,832 x^{3}+5,035 \times 10^{-4}(1-2 x) T
$$

is difficult to obtain with precision while growing the ternary alloys and the transverse effective masse in superlattice is two orders higher than in the alloy. Thus the tunnel length is small in the superlattice (A. Nafidi et al, 2011).

\section{The SL2: $\mathrm{HgTe}\left(d_{1}=18 \mathrm{~nm}\right) / \mathrm{CdTe}\left(d_{2}=4.4 \mathrm{~nm}\right)$}

\subsection{Theoretical results and discussions}

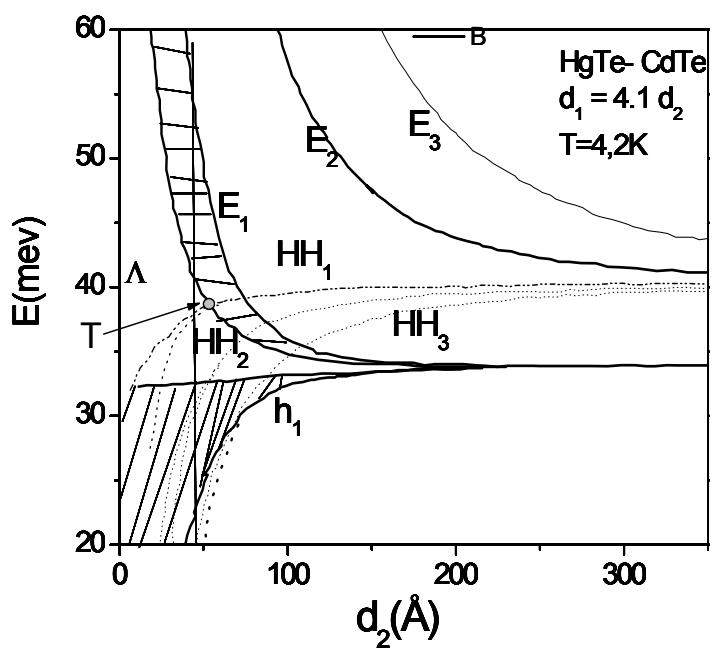

Fig. 8. Energy position and width of the conduction $\left(\mathrm{E}_{\mathrm{n}}\right)$, heavy-hole $\left(\mathrm{HH}_{\mathrm{n}}\right)$, and the first light-hole $\left(\mathrm{h}_{1}\right)$ subbands calculated at $4.2 \mathrm{~K}$ in the center $\Gamma$ of the first Brillion zone as a function of layer thickness $\mathrm{d}_{2}$ for $\mathrm{HgTe} / \mathrm{CdTe}$ superlattice with $\mathrm{d}_{1}=4.1 \mathrm{~d}_{2}$. T is the point of the transition semiconductor- semimetal 
The energy $E$ as a function of $d_{2}$, at $4.2 \mathrm{~K}$, in the center $\Gamma$ of the first Brillion zone and for $\mathrm{d}_{1}=$ $4.1 \mathrm{~d}_{2}$, is shown in Fig. 8. The case of our sample $\left(\mathrm{d}_{2}=44 \AA\right.$ ) is indicated by the vertical solid line. Here the cross-over of $\mathrm{E}_{1}$ and $\mathrm{HH}_{1}$ subbands occurs. $\mathrm{d}_{2}$ controls the superlattice band gap $\mathrm{Eg}=\mathrm{E}_{1}-\mathrm{HH}_{1}$. For weak $\mathrm{d}_{2}$ the sample is semiconductor with a strong coupling between the HgTe wells. At the point $\mathrm{T}\left(\mathrm{d}_{2}=53.7 \AA\right.$, $\left.\mathrm{E}=38.7 \mathrm{meV}\right)$ the gap goes to zero with the transition semiconductor- semimetal. When $d_{2}$ increases, $E_{1}$ and $h_{1}$ states drops in the energy gap $[0, \Lambda]$ and become interface state with energy $E_{I}=34 \mathrm{meV}$ for infinite $\mathrm{d}_{2}$. Then the superlattice has the tendency to become a layer group of isolated HgTe wells and thus assumes a semimetallic character. The ratio $\mathrm{d}_{1} / \mathrm{d}_{2}$ governs the width of superlattice subbands (i.e. the electron effective mass). A big $d_{1} / d_{2}$, as in our case, moves away the material from the two-dimensional behavior.

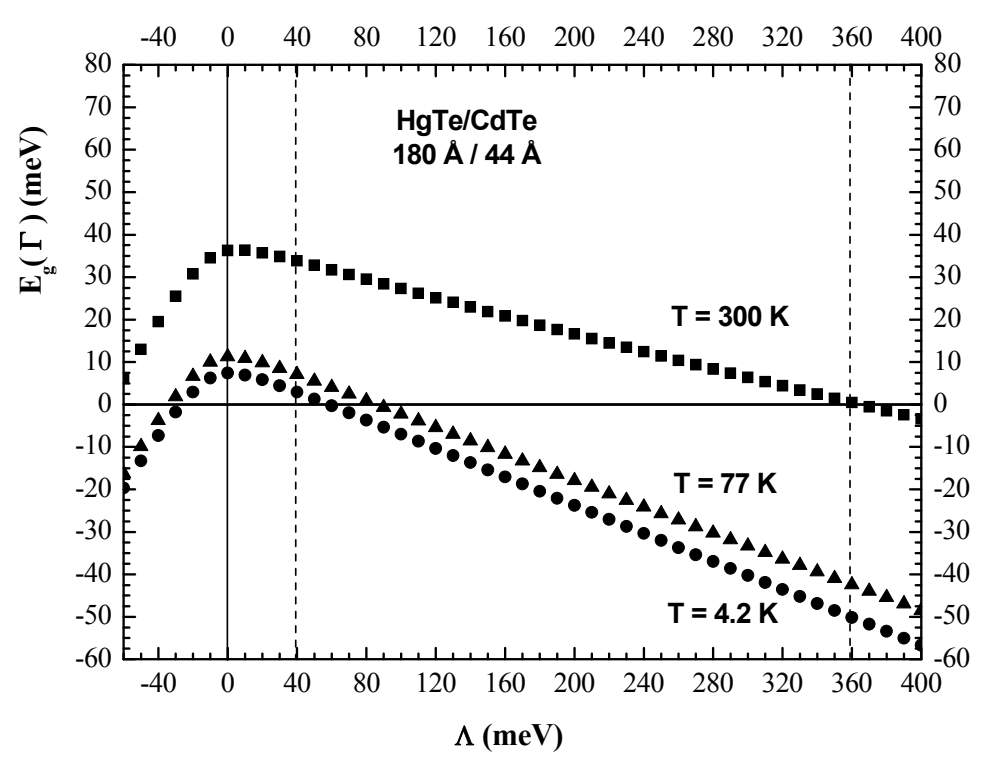

Fig. 9. The band gap $\mathrm{E}_{\mathrm{g}}(\Gamma)$, at the center $\Gamma$ of the first Brillion zone, as function of temperature and valence band offset $\Lambda$ between heavy holes bands edges of $\mathrm{HgTe}$ and $\mathrm{CdTe}$ for the investigated $\mathrm{HgTe} / \mathrm{CdTe}$ superlattice

In Fig. 9 we can see that the band gap $\mathrm{E}_{\mathrm{g}}(\Gamma)$ increases, presents a maximum at $0 \mathrm{meV}$ and decreases when the valence band offset $\Lambda$ between heavy hole band edges of $\mathrm{HgTe}$ and CdTe increase. For each $\Lambda, \mathrm{E}_{\mathrm{g}}(\Gamma)$ increases with $\mathrm{T}$. Our chosen value of $40 \mathrm{meV}$ is indicated by a vertical dashed line. This offset agrees well with our experimental results contrary to 0 meV used by (Bastard, 1982) and $360 \mathrm{meV}$ given by (Johnson et al, 1988). The later offset give a zero gap whereas, in intrinsic regime, our measured $E_{g}=38 \mathrm{meV}$ agree with calculated $\mathrm{E}_{\mathrm{g}}(\Gamma, 300 \mathrm{~K})=34 \mathrm{meV}$. 


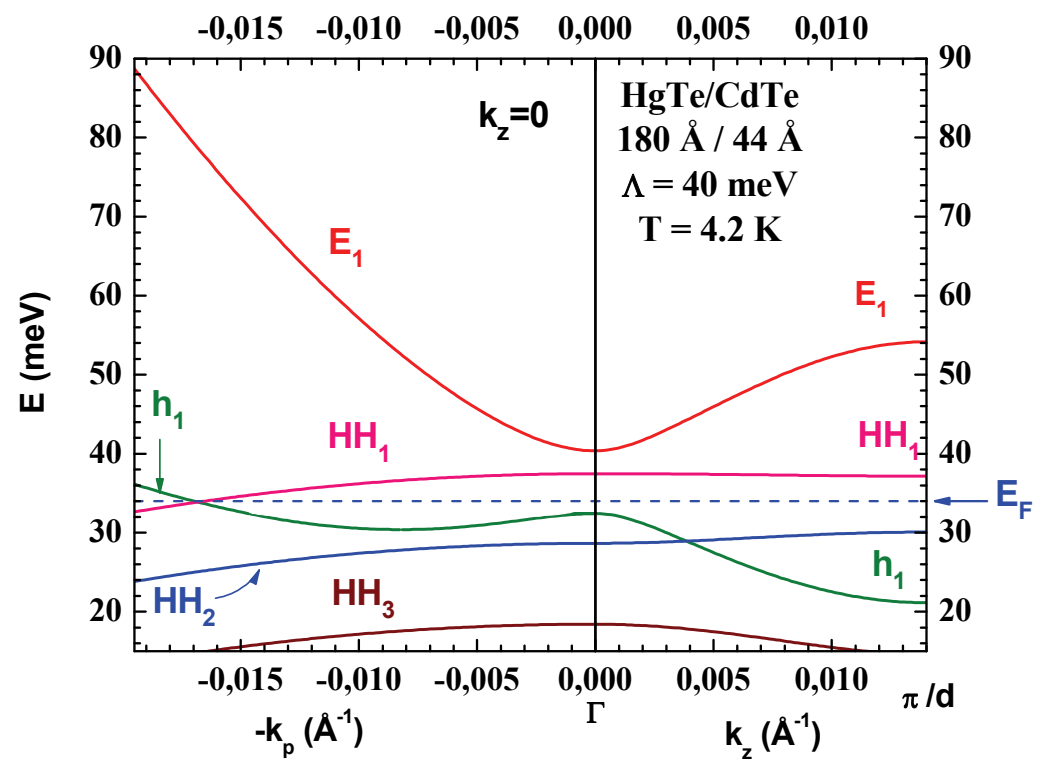

Fig. 10. Calculated bands along the wave vector $k_{z}(a)$ and in plane $k_{p}\left(k_{x}, k_{y}\right)(b)$, of the $\mathrm{HgTe} / \mathrm{CdTe}$ superlattice at $4.2 \mathrm{~K} . \mathrm{E}_{\mathrm{F}}$ is the energy of Fermi level

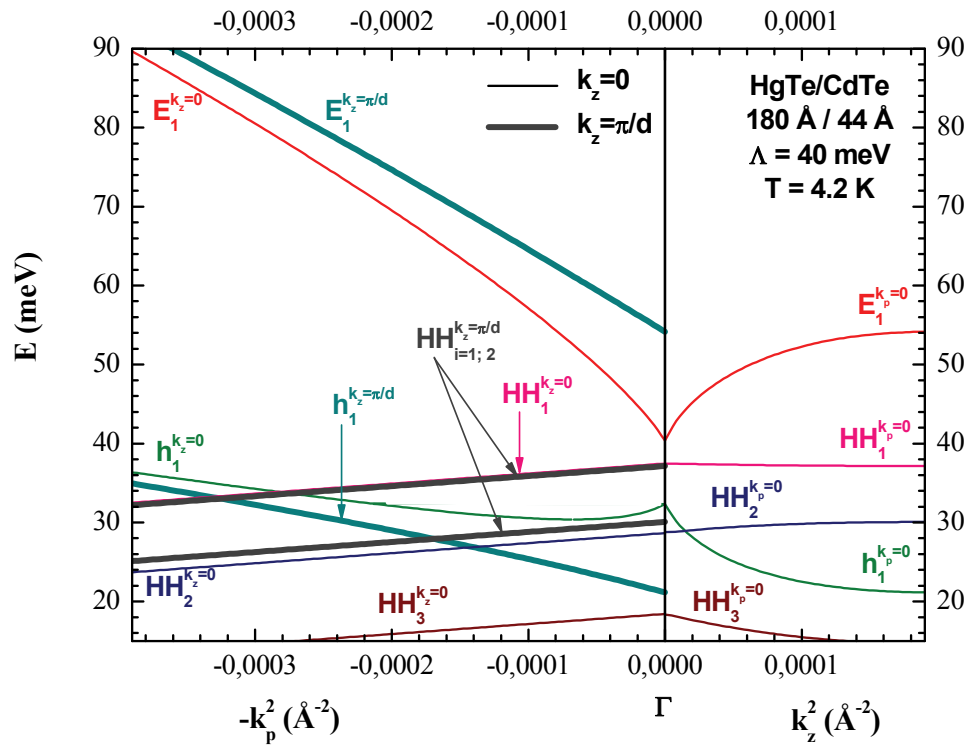

Fig. 11. Calculated bands as a function of $\mathrm{k}_{\mathrm{z}}^{2}$ and $\mathrm{k}_{\mathrm{p}}^{2}$ of the $\mathrm{HgTe} / \mathrm{CdTe}$ superlattice at $4.2 \mathrm{~K}$ 


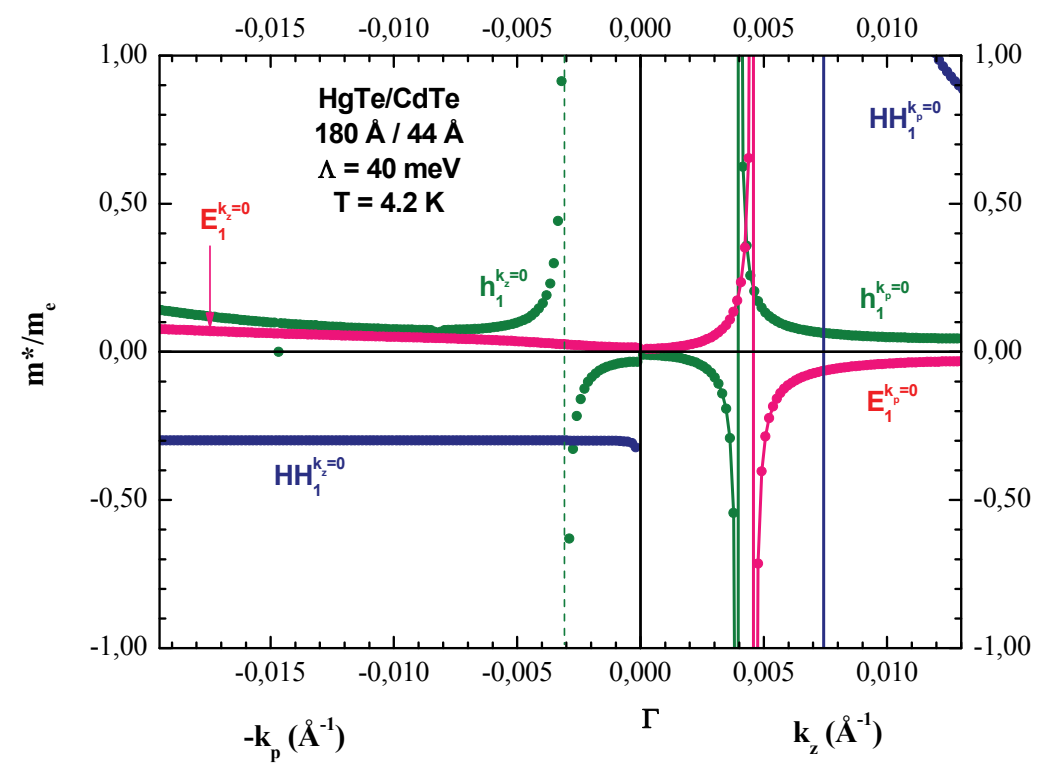

Fig. 12. Calculated relative effective mass bands along the wave vector $k_{z}$ and in plane $k_{p}$ of the $\mathrm{HgTe} / \mathrm{CdTe}$ superlattice at $4.2 \mathrm{~K}$

Fig. 10. show the spectra of energy $E\left(k_{z}\right)$ and $E\left(k_{p}\right)$, respectively, in the direction of growth and in plane of the superlattice at $4.2 \mathrm{~K}$. Along $\mathrm{E}\left(\mathrm{k}_{\mathrm{p}}\right), \mathrm{E}_{1}$ and $\mathrm{h}_{1}$ increase with $\mathrm{kp}$ whereas $\mathrm{HH}_{\mathrm{n}}$ decreases. This yields to a anti-crossing of $\mathrm{HH}_{1}$ and $\mathrm{h}_{1}$ at $\mathrm{k}_{\mathrm{p}}=0.0168 \AA^{-1}$. The gap is $\mathrm{E}_{\mathrm{g}}(\Gamma, 4.2 \mathrm{~K})=3 \mathrm{meV}$. Note that $\mathrm{E}_{\mathrm{F}}(4.2 \mathrm{~K})=34 \mathrm{meV}=\mathrm{E}_{\mathrm{I}}$ and then the conduction is assumed by heavy and light holes. As seen in Fig. 11., along $k_{z}$ and $k_{p}$ the light particles (electrons and light holes) subbands are not parabolic whereas the heavy hole subbands are parabolic.

For an anisotropic medium, such as the $\mathrm{HgTe} / \mathrm{CdTe}$ superlattices, the effective mass is a tensor and its elements along $\mu$ and $\nu$ directions are given by the following expression [Kittel, 2001]:

$$
\left(\frac{1}{\mathrm{~m}^{*}}\right)_{\mu v}=\frac{1}{\hbar^{2}} \frac{\partial^{2} \mathrm{E}_{\mathrm{k}_{\mu v}}}{\partial \mathrm{k}_{\mu} \partial \mathrm{k}_{v}}
$$

By carrying out second derivative of the energy $\mathrm{E}_{1}, \mathrm{~h}_{1}$ and $\mathrm{HH}_{1}$ along $\mathrm{k}_{\mathrm{z}}$ and $\mathrm{k}_{\mathrm{p}}$ in Fig. 10 we calculated the effective mass bands in Fig. 12. Along $k_{p}$, the effective mass of heavy holes $m^{*}{ }_{H H 1}=-0.300 m_{0}$ and the effective mass of electrons $m^{*}{ }_{E 1}$ increases from $0.011 m_{0}$ to 0.080 $\mathrm{m}_{0}$ whereas the effective mass of the light holes $h_{1}$ deceases from $0.140 \mathrm{~m}_{0}$ by half to a minimum of $0.074 \mathrm{~m}_{0}$. After it increases and diverges at $\mathrm{k}_{\mathrm{p}}=0.003 \AA^{-1}$ assuming a electronic conduction. After it increases to $-0.034 \mathrm{~m}^{0}$ at the center $\Gamma$ of the first Brillion zone assuming a light hole conduction.

Using the same procedure as for the SL1 we get the temperature dependence of the band gap $E_{g}$, in the center $\Gamma$ of the first Brillouin zone in Fig. 13. Note that $E_{g}$ increases from 3.6 
$\mathrm{meV}$ at $4.2 \mathrm{~K}$ to $34 \mathrm{meV}$ at $300 \mathrm{~K}$. In the investigated temperature range, the detection cutoff wave length verify : $40 \mu \mathrm{m} \leq \lambda_{\mathrm{c}} \leq 500 \mu \mathrm{m}$. This situates our sample as a far infrared detector.

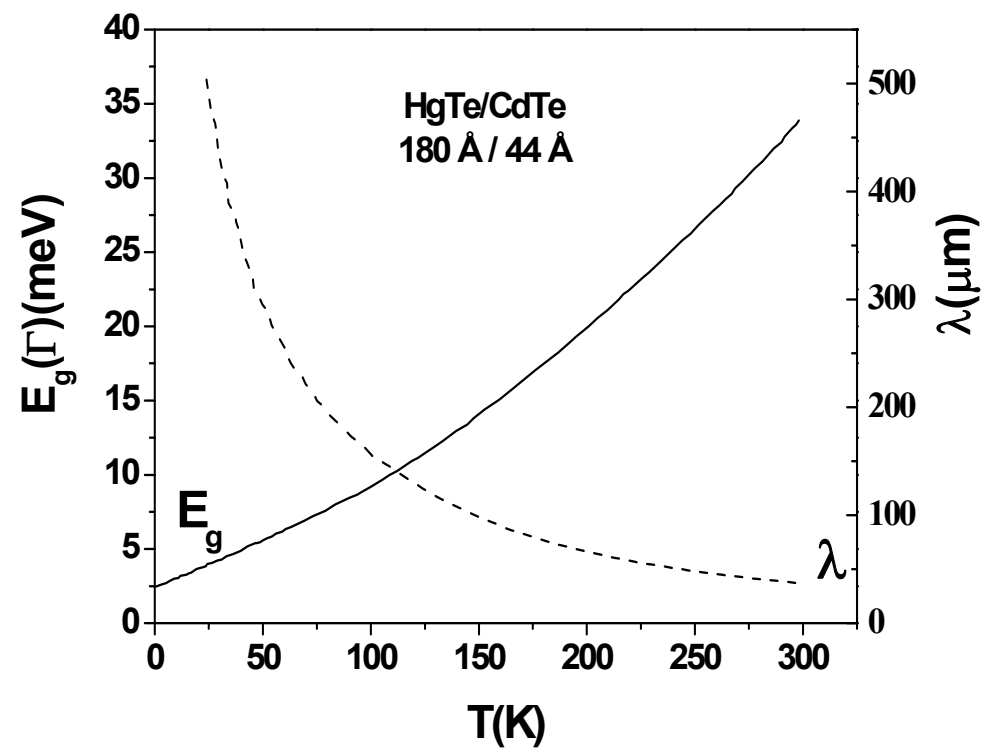

Fig. 13. Temperature dependence of the band gap $E_{g}$ and cut-off wavelength, at the center $\Gamma$ of the first Brillion zone, in the investigated $\mathrm{HgTe} / \mathrm{CdTe}$ superlattice

\subsection{Experimental results and discussions}

The transverse magnetoresistance $\rho / \rho_{0}$, in Fig. 14, follows the two-dimensional (2D) dependence with manifestation of the beginning of the Shubnikov-de Haas oscillations (with a weak $\left.\left[\left(\Delta \rho / \rho_{0}\right) \max =0.2\right]\right)$. However, over the entire investigated magnetic field range, a non-vanishing magnetoresistance is observed when the field is parallel to the plane. This can be due to the inter-diffusion between HgTe well (big $\mathrm{d}_{1} / \mathrm{d}_{2}$ and small $\mathrm{d}_{2}$ ) and/or to the widening of carriers subbands under the influence of the magnetic field along $E\left(k_{\mathrm{p}}\right)$. While the Hall voltage $V_{H}$ goes to zero at this configuration in Fig. 15. This suggests quasi two-dimensional conductivity behavior (between three-dimensional (3D) and twodimensional (2D).

At low temperature, the sample exhibits $\mathrm{p}$ type conductivity with a hole mobility $\mu_{\mathrm{p}} \approx 900$ $\mathrm{cm}^{2} /$ V.s. As seen in Fig. 16 (b), a reversal of the sign of the weak-field Hall constant $R_{H}$ occurs at $25 \mathrm{~K}$. It may be attributed to trapping of carrier charges in intrinsic state $\mathrm{E}_{\mathrm{I}}$. Such a reversal of the sign of the Hall voltage may be inferred by the existence of at least two types of carriers, which suggests a semimetallic character of the conduction mechanism.

Fallowing the relaxation time resolution of the Boltzmann equation (Seeger b, 2002) (Kittel, 2001) the Hall constant at weak magnetic field in Fig. 16 (b) is described by the well knows formula: 


$$
\left(R_{H}\right)_{w}=\frac{1}{|e|} \frac{p-n b^{2}}{(p+n b)^{2}} \quad \text { with } \quad b=\frac{\mu_{n}}{\mu_{p}}
$$

At low temperature in the saturation regime:

$$
\left(\mathrm{R}_{\mathrm{H}}\right)_{\mathrm{sat}}=\frac{1}{|\mathrm{e}| \mathrm{p}}
$$

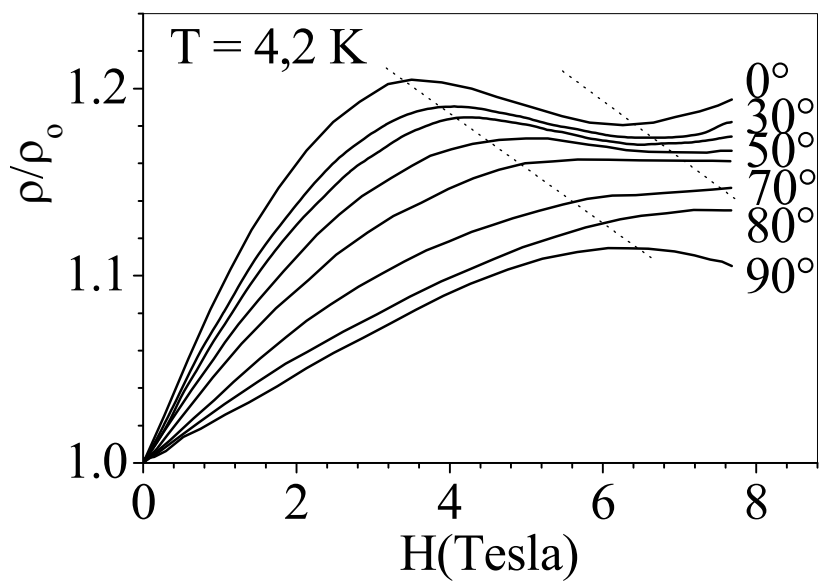

Fig. 14. Transverse magnetoresistance of the sample, at various angles between the magnetic field and the normal to the $\mathrm{HgTe} / \mathrm{CdTe}$ superlattice surface, at $4.2 \mathrm{~K}$

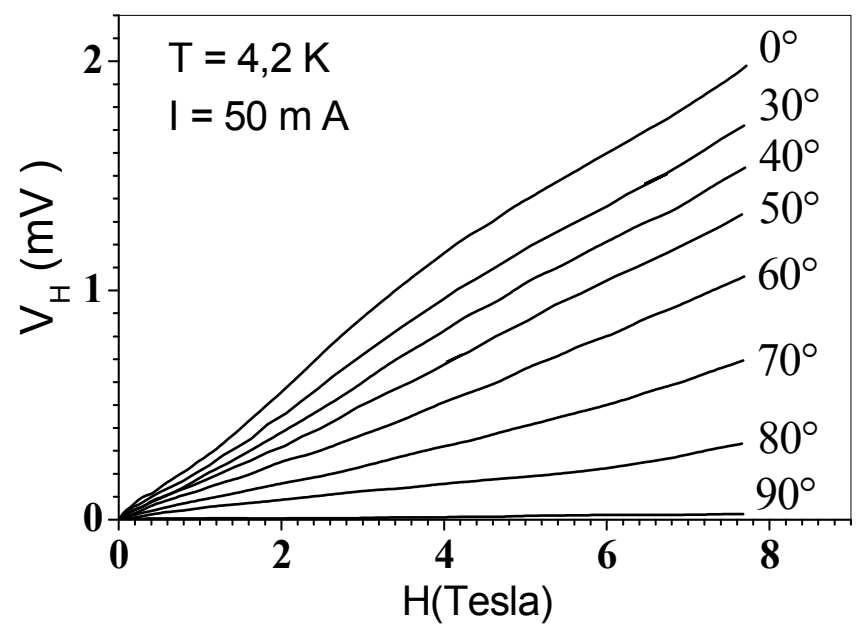

Fig. 15. Field dependence of Hall voltage, at various angles between the magnetic field and the normal to the $\mathrm{HgTe} / \mathrm{CdTe}$ superlattice surface, at $4.2 \mathrm{~K}$ 


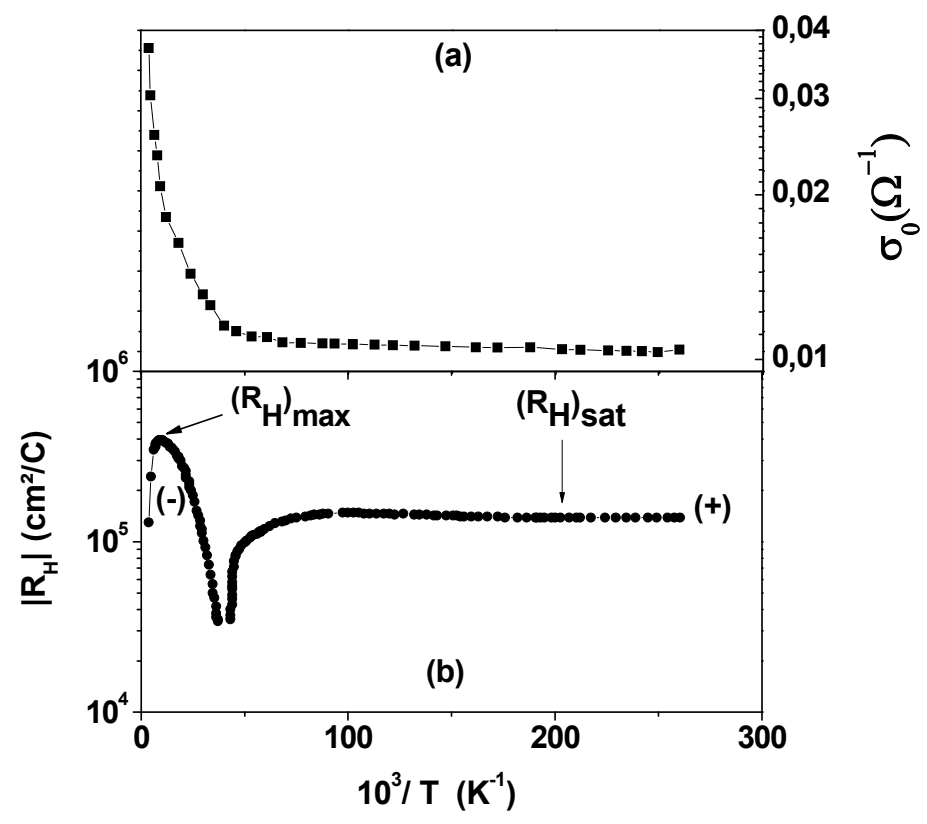

Fig. 16. Temperature dependence of the conductivity (a) and weak-field Hall coefficient (b), in the investigated $\mathrm{HgTe} / \mathrm{CdTe}$ superlattice

Near the intrinsic regime, the maximum of the Hall constant is given by:

$$
\left(\mathrm{R}_{\mathrm{H}}\right)_{\max }=\frac{(\mathrm{b}-1)^{2}}{|\mathrm{e}| 4 \mathrm{bp}}
$$

This results in the ratio:

$$
\frac{4\left(\mathrm{R}_{\mathrm{H}}\right)_{\max }}{\left(\mathrm{R}_{\mathrm{H}}\right)_{\text {sat }}}=\frac{(\mathrm{b}-1)^{2}}{\mathrm{~b}} \approx \mathrm{b}=32
$$

Eq. (15) implies for $\mathrm{R}_{\mathrm{H}}=0$ :

$$
\mathrm{b}^{2}=1024=\frac{\mathrm{p}}{\mathrm{n}}
$$

This implies an electron mobility of $\mu_{\mathrm{n}}=3 \times 10^{4} \mathrm{~cm}^{2} /$ V.s. Such a low value of the electron mobility can be connected, from the one side, with the superlattice electron effective mass which is much higher than that of the bulk material (Nafidi et al, 2011) and, from the other side, with different types of imperfections of the investigated structures, including strong compensation.

From Eq. (3) we have:

$$
\frac{2}{3} \mathrm{P}^{2} \hbar^{2} \mathrm{k}_{\mathrm{F}}^{2}=\mathrm{E}_{\mathrm{F}}\left(\mathrm{E}_{\mathrm{F}}-\mathrm{E}_{\mathrm{g}}\right)
$$


We extract the Fermi level energy:

$$
\mathrm{E}_{\mathrm{F}}=\frac{\mathrm{E}_{\mathrm{g}}}{2} \pm \sqrt{\left(\frac{\mathrm{E}_{\mathrm{g}}}{2}\right)^{2}+\frac{2}{3} \mathrm{P}^{2} \hbar^{2} \mathrm{k}_{\mathrm{F}}^{2}}
$$

Where the Fermi wave vector is $k_{F}(3 D)=\left(3 \Pi^{2} p\right)^{1 / 3}$ and $k_{F}(2 D)=(2 \pi p)^{1 / 2}$, respectively for three-dimensional and two-dimensional motion of carriers charges. Here we have chosen the minus sign because the sample is p-type at low temperature. Fig. 10 shows that the band gap and the 3D Fermi level energy increase with temperature whereas 2D Fermi level energy and $\mathrm{HH}_{\mathrm{i}}$ bands energy remain constant. In all cases the conductivity is assumed by light and heavy holes. So we have quasi-two-dimensional and semimetallic conductivity. In table1 bellow we summarized the electronic transport parameters in the investigated two superlattices.

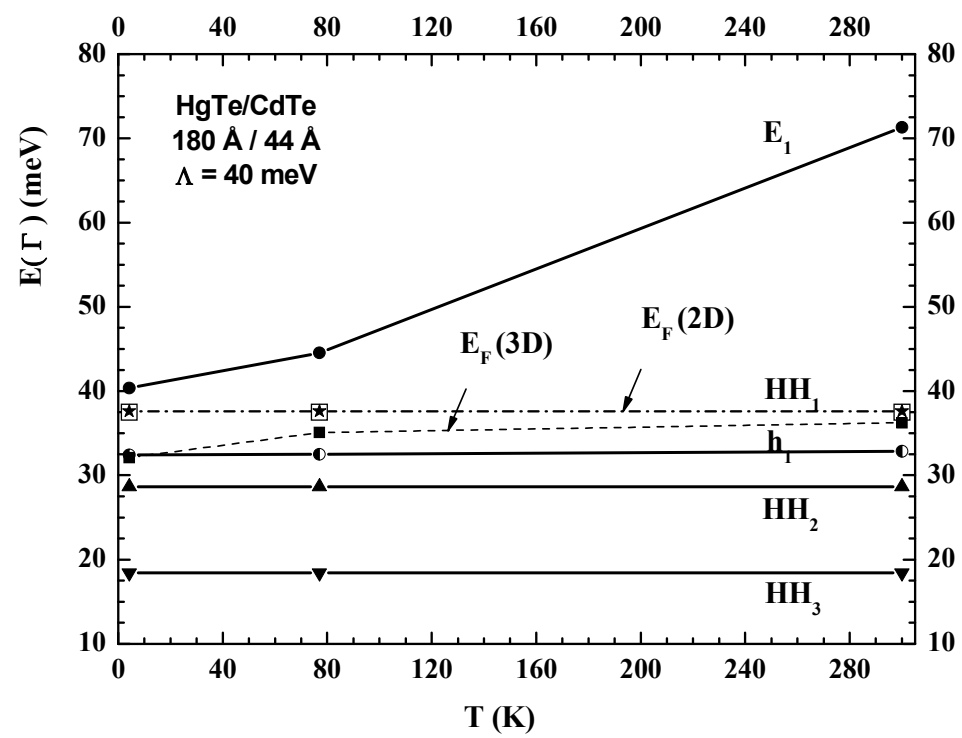

Fig. 17. Temperature dependence of the energy for the three-dimensional (3D) and twodimensional (2D) Fermi levels in the investigated HgTe/CdTe superlattice

\begin{tabular}{|c|c|c|c|c|c|}
\hline & $\begin{array}{c}\mathrm{E}_{\mathrm{g}}(\mathrm{meV}) \\
\text { Theory 4.2 } \mathrm{K}\end{array}$ & $\begin{array}{c}\lambda_{\mathrm{c}}(\mu \mathrm{m}) \\
\text { Theory } 4.2 \mathrm{~K}\end{array}$ & $\begin{array}{c}\mathrm{E}_{\mathrm{F}}(\mathrm{meV}) \\
\text { Exper. 4.2 K }\end{array}$ & $\begin{array}{c}\mathrm{p}\left(\mathrm{cm}^{-2}\right) \\
\text { Hall ef. 4.2K }\end{array}$ & $\begin{array}{c}\mu_{\mathrm{p}}\left(\mathrm{cm}^{2} / \mathrm{Vs}\right) \\
\text { Hall ef. 4.2K }\end{array}$ \\
\hline $\begin{array}{c}\text { SL1 } \\
\text { (p-type) }\end{array}$ & 111 & $11(\mathrm{MIR})$ & $14(2 \mathrm{D})$ & $1.84 \times 10^{12}$ & 8200 \\
\hline $\begin{array}{c}\text { SL2 } \\
\text { (p-type) }\end{array}$ & 3.6 & $464(\mathrm{FIR})$ & $\begin{array}{c}32(3 \mathrm{D}) \\
38(2 \mathrm{D})\end{array}$ & $4.51 \times 10^{13}$ & 900 \\
\hline
\end{tabular}

Table 1. Electronic transport parameters in the investigated two $\mathrm{HgTe} / \mathrm{CdTe}$ superlattices 


\section{Conclusions}

The fundamental main ideas of this work are:

- HgTe is a zero gap semiconductor (or semimetal) when it is sandwiched between the wide gap semiconductor $\mathrm{CdTe}(1.6 \mathrm{eV}$ at $4.2 \mathrm{~K})$ layers yield to a narrow gap $\mathrm{HgTe} / \mathrm{CdTe}$ superlattice which is the key of an infrared detector.

- Before growing our two superlattices, we calculated the bands structures $E\left(d_{2}\right)$ and the gap for each ratio thickness $d_{1} / d_{2}$. After we choose the SL1 in the semiconductor conductivity zone and the SL2 at the transition semiconductor-semimetal

We reported here remarkable correlations between calculated bands structures and magnetotransport properties in two $\mathrm{HgTe} / \mathrm{CdTe}$ nanostructures superlattices SLi (i=1 and 2). Our calculations of the specters of energy $\mathrm{E}\left(\mathrm{d}_{2}\right), \mathrm{E}\left(\mathrm{k}_{\mathrm{z}}\right)$ and $\mathrm{E}\left(\mathrm{k}_{\mathrm{p}}\right)$, respectively, in the direction of growth and in plane of the superlattice; were performed in the envelope function formalism. In the SL1, the formalism predicts that the system is semiconductor, for our HgTe to CdTe thickness ratio $d_{1} / d_{2}=1.87$, when $d_{2}<14 \mathrm{~nm}$. In our case, $d_{2}=3 \mathrm{~nm}$ and $E_{g}(\Gamma, 4.2 \mathrm{~K})=111$ $\mathrm{meV}$. In spite of it, the sample exhibits the features typical for the semiconductor type $p$ conduction mechanism. In the used temperature range, this simple is a medium-infrared detector, narrow gap and two-dimensional p-type semiconductor.

In the SL2, the formalism predicts that the system is semimetallic, for our HgTe to CdTe thickness ratio $d_{1} / d_{2}=4.1$ for $d_{2} \geq 3 \mathrm{~nm}$. In our case $d_{2}=4.4 \mathrm{~nm}$ and the gap $E_{g}(\Gamma, 4.2 \mathrm{~K})=3$ $\mathrm{meV}$ corresponding to thermal energy necessary to change the sign of $\mathrm{R}_{\mathrm{H}}(\mathrm{T})$. In intrinsic regime, the measurements indicates $\mathrm{E}_{\mathrm{g}} \approx 38 \mathrm{meV}$ in good agreement with calculated $\mathrm{E}_{\mathrm{g}}(\Gamma$, $300 \mathrm{~K})=34 \mathrm{meV}=\mathrm{E}_{\mathrm{F}}(4.2 \mathrm{~K})=\mathrm{E}_{\mathrm{I}}$. In spite of it, the sample exhibits the features typical for the semimetallic conduction mechanism, which agree well with the overlap between carrier subbands along $\mathrm{E}\left(\mathrm{k}_{\mathrm{p}}\right)$ with a quasi-two-dimensional behavior and is a far-infrared detector. Our HgTe/CdTe superlattices SL1 and SL2 are a stable alternative for application in infrared optoelectronic devices than the alloys $\mathrm{Hg}_{0.8} \mathrm{Cd}_{0.2} \mathrm{Te}$ and $\mathrm{Hg}_{0.66} \mathrm{Cd}_{0.34} \mathrm{Te}$ respectively. The theoretical and magnetotransport parameters are in good agreement. Measurements performed by us on others' samples indicate an improvement of quality of the material manifested by higher mobility.

\section{Acknowledgements}

This work is supported by research budget of University Ibn Zohr in Agadir. We thank Li Rukang from the School of Chemistry, University of Birmingham, for verifying our X-ray diffraction Rietveld refinements.

\section{References}

Arch, D. K.; Faurie, J. P.; Staudenmann, J.L.; Hibbs-Brenner, M. \& Chow, P. (1986). Interdiffusion in HgTe-CdTe superlattices. J. Vac. Sci. Technol. A, Vol, 4, (1986) (2101-2106), DOI: 10.1116/1.574035.

Bastard, G. (1981). Superlattice band structure in the envelope-function approximation. Phys. Rev. B, Vol,24, (November15, 1981) (5693-5697), DOI: 10.1103/PhysRevB.24.5693.

Bastard, G. (1982). Theoretical investigations of superlattice band structure in the envelopefunction approximation. Phys. Rev. B, Vol, 25, (June 15, 1982) (7584-7597), DOI: 10.1103/PhysRevB.24.5693. 
Cava, R. J. Science 247, pp. 656-662 (1990).

Dingle, R.; Gossard, A. C.; \& Wiegmann, W. (1975). Direct Observation of Superlattice Formation in a Semiconductor Heterostructure. Phys. Rev. Lett., Vol, 34, (May 26, 1975) (1327-1330), DOI:10.1103/PhysRevLett.34.1327.

El Abidi, A.; Nafidi, A.; Chaib, H.; El Kaaouachi, A.; Braigue, M.; Morghi, R.; EL Yakoubi, E.Y. \& M. d'Astuto, (2010). Application of the transition semiconductor semimetal in modulated nanostructures for communication as infrared optoelectronic device. Physica B: Physics of Condensed Matter, Vol, 405, (1 February 2010) (936-940), DOI:10.1016/j.physb.2009.10.019.

Esaki, L. \& Tsu, R. (1970). Superlattice and negative differential conductivity in Semiconductors. IBM J. Res. Development, Vol., 14, (Jan. 1970) (61-65), ISSN: 0018-8646.

Guldner, Y.; Bastard, G.; Vieren, J.P.; Voos, M.; Faurie, J.P. \& Million, A. (1984). Magnetooptics in a II-VI superlattice: HgTe-CdTe. Surface Science, Vol., 142, (1 July 1984) (593-597), DOI:10.1016/0039-6028(84)90367-4

Hansen, G. L.; Schmit, J. L. \& Casselman, T. N., (1982).Energy gap versus alloy composition and temperature in $\mathrm{Hg}_{1-\mathrm{x}} \mathrm{Cd}_{\mathrm{x}} \mathrm{Te}$. J. Appl. Phys, Vol., 53, (10 June 1982) (7099-7101), DOI:10.1063/1.330018.

Johnson, N. F.; Hui, P. M. \& Ehrenreich, H. (1988). Valence-Band-Offset Controversy in HgTe/CdTe Superlattices: A Possible Resolution. Phys. Rev. Lett., Vol, 61, (24 October 1988) (1993-1995), DOI: 10.1103/PhysRevLett.61.1993

Kane, E. (1957). Band structure of indium antimonide. Journal of Physics and Chemistry of Solids, Vol. 1, Issue 4,( January 1957) (249-261). DOI: 10.1016/0022-3697(57)90013-6

Kittel, C. (2001). Introduction to solid stat physics, 3d edition, John Wiley and Sons, Inc, New York, 333.

Nafidi, Ab.; El Kaaouachi, A.; Sahsah, H.; Nafidi, Ah. (2002). Band structure and magnetotransport in $\mathrm{HgTe} / \mathrm{CdTe}$ superlattice. Book of the International Conference on Theoretical Physics (HT 2002), pp. 274-275, ISBN 3-7643-2433-3, , 22-27 July, Paris, France Birkhäuser Verlag, Germany.

Nafidi, A.; El Kaaouachi, A.; Nafidi, Ah.; Faurie, J.P.; Million, A.; Piaguet, J. (2002). Some Transport Properties of HgTe/CdTe Superlattices. Physica status solidi (b), Vol. 229, (January 2002) (573-576), DOI: 10.1002/1521.

Nafidi, Ab.; EL Abidi, A.; El Kaaouachi, A.; \& Nafidi, Ah. (2004). Electronic Band Structure and New Magneto-transport Properties in p-type Semiconductor Medium-infrared HgTe / CdTe Superlattice. 27th International Conference on the Physics of Semiconductors - ICPS-27, AIP Conference Proceedings, Vol. 772, pp. 1001-1002, DOI:10.1063/1.1994448, July 26-30, 2004, in Flagstaff, Arizona, USA.

Nafidi, A.; El Abidi, A. \& El Kaaouachi, A. (2006). Seebeck and Shubnikov-de Haas Effects in a Two-Dimensional p-type HgTe/CdTe Superlattice. 24th International Conference on Low Temperature Physics - LT24 , AIP Conference Proceedings Vol. 850,pp. 1359-1360, ISBN: 0735403473, 10 - 17 Aug 2005, Orlando, FL, USA.

Nafidi A., Bouallal A., El Kaaouachi B., A. and Chaib, H., IEEE Transactions on Applied Superconductivity. Vol 17 , NO 02, pp 2969-2972, (2007).

Nafidi A., Bouallal B., El Kaaouachi A., Chaib H. and Sahsah H., Advance in Cryogenic Engineering, Transactions of the International Cryogenic Materials conference-ICMC, American Institute of Physics, AIP CP986, Vol.54, pp.551-558, (2008).

Nafidi, A., et al, (2011) to be published 
Sakaki, H.; Chang, L. L.; Sai-Halasz, G. A.; Chang, C. A. \& Esaki, L. (1978). Two-dimensional electronic structure in InAs-GaSb superlattices. Solid State Communications, Vol. 26, (June 1978) (589-592), DOI:10.1016/0038-1098(78)90770-6

Seeger, K. a (2002). Semiconductor physics: an introduction, Springer, ISBN: 9783540438137, Edition Number: 8, chap.4, p.121.

Seeger, K. b (2002). Semiconductor physics: an introduction, Springer, ISBN: 9783540438137, Edition Number: 8, chap.6, p.159.

Tokura, Y. Takagi, H and Uchida, S. Nature 337, pp. 345-347 (1989).

Tuchendler, J.; Grynberg, M.; Couder, Y.; Thomé, H. \& Le Toullec R. (1973). Submillimeter Cyclotron Resonance and Related Phenomena in HgTe. Phys. Rev. B, Vol., 8, (15 October 1973) (3884-3894), DOI: 10.1103/PhysRevB.8.3884.

Weiler, M. H. (1981). Magnetooptical Properties of Hg1-xCdxTe Alloys, In: semiconductors and Semimetals, Vol., 16, Willardson, R. K. \& Beer, A. C. (Ed), (119-191) (Academic, New York), ISBN: 9780127521169 


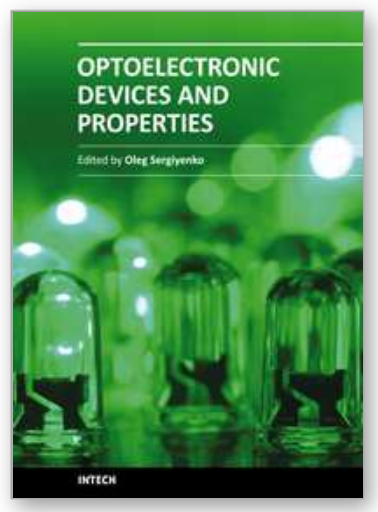

\section{Optoelectronic Devices and Properties}

Edited by Prof. Oleg Sergiyenko

ISBN 978-953-307-204-3

Hard cover, 660 pages

Publisher InTech

Published online 19, April, 2011

Published in print edition April, 2011

Optoelectronic devices impact many areas of society, from simple household appliances and multimedia systems to communications, computing, spatial scanning, optical monitoring, 3D measurements and medical instruments. This is the most complete book about optoelectromechanic systems and semiconductor optoelectronic devices; it provides an accessible, well-organized overview of optoelectronic devices and properties that emphasizes basic principles.

\section{How to reference}

In order to correctly reference this scholarly work, feel free to copy and paste the following:

Abdelhakim Nafidi (2011). Band Structure and Magneto- Transport Properties in II-IV Nanostructures Semiconductors - Application to Infrared Detector Superlattices, Optoelectronic Devices and Properties, Prof. Oleg Sergiyenko (Ed.), ISBN: 978-953-307-204-3, InTech, Available from:

http://www.intechopen.com/books/optoelectronic-devices-and-properties/band-structure-and-magnetotransport-properties-in-ii-iv-nanostructures-semiconductors-application-t

\section{INTECH}

open science | open minds

\section{InTech Europe}

University Campus STeP Ri

Slavka Krautzeka 83/A

51000 Rijeka, Croatia

Phone: +385 (51) 770447

Fax: +385 (51) 686166

www.intechopen.com

\section{InTech China}

Unit 405, Office Block, Hotel Equatorial Shanghai

No.65, Yan An Road (West), Shanghai, 200040, China

中国上海市延安西路65号上海国际贵都大饭店办公楼 405 单元

Phone: +86-21-62489820

Fax: $+86-21-62489821$ 
(C) 2011 The Author(s). Licensee IntechOpen. This chapter is distributed under the terms of the Creative Commons Attribution-NonCommercialShareAlike-3.0 License, which permits use, distribution and reproduction for non-commercial purposes, provided the original is properly cited and derivative works building on this content are distributed under the same license. 